\title{
Modulation of the transforming growth factor-B1-induced Smad phosphorylation by the extracellular matrix receptor B1-integrin
}

\author{
HIROSHI HAMAJIMA ${ }^{1}$, IWATA OZAKI ${ }^{1,2}$, HAO ZHANG $^{1,3}$, SHINJI IWANE $^{1}$, \\ YASUNORI KAWAGUCHI ${ }^{1}$, YUICHIRO EGUCHI ${ }^{1}$, SACHIKO MATSUHASHI ${ }^{1}$, \\ TOSHIHIKO MIZUTA $^{1}$, KOICHI MATSUZAKI ${ }^{4}$ and KAZUMA FUJIMOTO ${ }^{1}$
}

\begin{abstract}
${ }^{1}$ Department of Internal Medicine, Saga Medical School, Saga University; ${ }^{2}$ Health Administration Center, Saga Medical School, Saga University, 5-1-1 Nabeshima, Saga 849-8501, Japan; ${ }^{3}$ Department of Surgery, The First Affiliated Hospital, China Medical University, Shenyang 110001, P.R. China; ${ }^{4}$ Department of Gastroenterology and Hepatology, Kansai Medical University, 10-15 Fumizonocho, Moriguchi, Osaka 570-8507, Japan
\end{abstract}

Received June 29, 2009; Accepted September 2, 2009

DOI: 10.3892/ijo_00000463

\begin{abstract}
Integrins, heterodimeric receptors for the extracellular matrix, are known to modulate transforming growth factor- $\beta 1$ (TGF- $\beta 1$ )-mediated cell behavior. However, the interplay between $\beta 1$-integrin and Smad signaling, regulated by TGF- $\beta 1$, is not clearly understood. This study focuses on the alterations of the regulatory Smads (R-Smads) by TGF- $B 1$ in $\beta 1$-integrin-transfected HepG2 cells. The phosphorylation at the C-terminal site of R-Smads by TGF- 31 was impaired in the B1-integrin-transfected cells. However, the R-Smads were constitutively phosphorylated at the linker region in a MAP kinase-dependent manner. Furthermore, the expression of a mutant Smad3, that lacks the phosphorylation sites in the linker region, restored the TGF- $\beta 1$-induced Smad transcriptional activity. These results suggest that $\beta 1$-integrin impairs the TGF- 31 -mediated signals through the altered phosphorylation of the R-Smads.
\end{abstract}

\section{Introduction}

Hepatocellular carcinoma (HCC) is ranked as the fifth most common malignant neoplasm in the world and it is closely associated with hepatitis $\mathrm{B}$ and/or $\mathrm{C}$ viral infections $(1,2)$. The chronic infection that is related to the hepatitis viruses causes persistent inflammation of the liver which results in cirrhosis, a condition characterized by an excessive accumulation of the extracellular matrices. Liver cirrhosis is associated with the development of HCC in both clinical observations and in experimental animal models $(1,3,4)$.

Correspondence to: Dr Iwata Ozaki, Department of Internal Medicine, Health Administration Center, Saga Medical School, Saga University, 5-1-1 Nabeshima, Saga 849-8501, Japan

E-mail: ozaki@cc.saga-u.ac.jp

Key words: transforming growth factor- $\$ 1$, Smad, phosphorylation, ß1-integrin, hepatocellular carcinoma
Transforming growth factor- $\beta 1$ (TGF- $\beta 1)$ is a potent stimulator of the extracellular matrix $(\mathrm{ECM})$ production in the liver and it also participates in the development of liver cirrhosis (5). TGF-ß1 plays a dual role in carcinogenesis. TGF- 31 inhibits the proliferation of the epithelial, the endothelial and the hematopoietic cells and therefore acts as a tumor suppressor (6). TGF- $\$ 1$ induces an epithelial-tomesenchymal transition (EMT) in cells which increases tumor invasiveness resulting in the promotion of tumor metastasis (7). TGF- $\beta$ transduces the signals from its receptor to the nucleus through the Smad proteins. The phosphorylation of the receptors and the intracellular signaling molecules play an important role in most of the signal transduction pathways. The activated TGF- $\beta$ type I receptor (TßRI) phosphorylates the C-terminal SSXS motif of the receptor-regulated Smads (R-Smads), such as Smad2 and Smad3. The phosphorylated $\mathrm{R}$-Smads can oligomerize with Smad4 to form a functional complex which is translocated to the nucleus, where it induces transcriptional induction or repression of the target genes $(8,9)$. The linker region of the Smad proteins can be phosphorylated by several kinases, including the mitogen-activated protein (MAP) kinases, such as the extracellular signal-regulated kinase (Erk), the c-Jun terminal kinase (JNK) and the p38 or the cyclin-dependent kinase (10-13). Several studies have demonstrated that the phosphorylation of the linker region of the R-Smads can cause an alteration of the TGF- $\beta 1$ signaling, thus resulting in the loss of the tumor suppressive function.

ß1-integrin is a major cell surface adhesion receptor that transmits signals from the ECM and that plays critical roles in growth, differentiation and carcinogenesis (14). B1-integrin can block the apoptosis that is induced by several reagents and can therefore function as a survival factor (15). The overexpression of $\beta 1$-integrin in HCC cells inhibits the growth suppressive signals and the apoptosis induced by TGF-ß1 (16). However, the mechanisms by which $\beta 1$-integrin modulates the TGF-ß1-induced Smad signaling is not known. Therefore, this study investigated the changes of the TGF- $\beta 1$-induced R-Smads phosphorylation by $\beta 1$-integrin in order to elucidate the mechanisms involved in the $\beta 1$-integrin-initiated alteration of the TGF- $ß$ signaling. 


\section{Materials and methods}

Cells and reagents. The HepG2 cells were obtained from the Japanese Cancer Research Resources (Osaka, Japan). The HepG2 cells overexpressing $\beta 1$-integrin, generated by stable transformation of a human B1A-integrin-enhanced green fluorescent protein (GFP) fusion gene, were described previously $(17,18)$. The cells were cultured and maintained in Dulbecco's modified Eagle's medium (DMEM; GibcoBRL, Gaithersburg, MD) containing $10 \%$ fetal calf serum (Gibco-BRL). The human recombinant TGF-ß1 was purchased from R\&D Systems (Minneapolis, MN), The PD98059 was purchased from Promega (Madison, WI), the SB203580 was purchased from Sigma (St. Louis, MO) and the SP600125 was purchased from Calbiochem (San Diego, CA).

Plasmids, transfection and the luciferase assay. The TGF-ßinducible luciferase-reporter plasmid containing the four Smad-binding elements (SBE4-Luc) (19) and the mammalian expression plasmids containing the mutations in the linker region of Smad2 and Smad3 (Smad2/EPSM and Smad3/ EPSM, respectively) (10) were obtained from Addgene (Cambridge, MA). The human $\$ 1 \mathrm{~A}$-integrin expression plasmid was described previously $(17,18)$. The Smaddependent transcriptions were detected by a luciferase assay according to the method described by the supplier (DualLuciferase Reporter Assay System; Promega). The luciferase expression plasmid, pRL-SV40 (Promega), was cotransfected to normalize any variations in the transfection efficiency. The HCC cells were seeded onto 6-well plates at a density of $1 \times 10^{5}$ cells/well without antibiotics and were then incubated at $37^{\circ} \mathrm{C}$ until $80 \%$ confluence was attained. The confluent cells were washed twice with OPTI-MEM I Reduced Medium (Life Technologies, Rockville, MD) prior to the addition of $2 \mathrm{ml}$ of OPTI-MEM I Reduced Medium containing $1 \mu \mathrm{g}$ of the target-gene reporter plasmid, $0.5 \mu \mathrm{g}$ of the pRL-SV40 luciferase plasmid and $15 \mu \mathrm{l}$ of the Lipofectamine 2000 reagent (Life Technologies). After $6 \mathrm{~h}$ of incubation, the medium was changed and the transfected cells were treated with the indicated doses of TGF- 31 . After $24 \mathrm{~h}$ of treatment, the cell were washed twice with PBS and carefully scraped into a $1 \mathrm{X}$ passive lysis buffer (Promega). The cell extracts were assayed immediately for luciferase activity using a Berthold Luminometer (MLR100 Micro Lumino Reader; Corona Electric, Ibaragi, Japan).

Antibodies. The rabbit polyclonal anti-Smad2, anti-Smad3, and anti-Histone $\mathrm{H} 1$ antibodies were purchased from Zymed Laboratories (San Francisco, CA). The antibodies against the phosphorylated form of the C-terminal region of Smad2 (Ser465/467) and Smad3 (Ser423/425), as well as the antibody against the phosphorylated form of the linker region of Smad2 (Ser245/250/255), were purchased from Cell Signaling (Boston, MA). The antibody against the phosphorylated form of the linker region of Smad3 was described previously $(11,12)$. The rabbit polyclonal anti-human $\beta$-actin antibody was purchased from Biomedical Technologies (Stoughton, MA).
Western blotting. The cultured HepG2 cells were treated with $2 \mathrm{ng} / \mathrm{ml}$ of TGF- $\beta 1$ and recovered at the indicated times. The cells were lysed with an extraction buffer containing $1 \mathrm{mM}$ phenylmethylsulfonyl fluoride (PMSF), $1 \mathrm{mM} \mathrm{Na} 3 \mathrm{VO}_{4}$ and $1 \mathrm{mM} \mathrm{NaF}$. The lysate was sonicated for $5 \mathrm{~min}$ at $4^{\circ} \mathrm{C}$ and was then clarified by centrifugation at $15,000 \mathrm{rpm}$ for $10 \mathrm{~min}$, after which the supernatant was collected. After the quantification of the protein with a protein assay kit (Bio-Rad, Hercules, CA), $40 \mu \mathrm{g}$ of the protein was mixed with a NuPAGE sample buffer (Novex, San Diego, CA). The mixture was separated by SDS polyacrylamide gel electrophoresis and transferred to a polyvinylamide difluoride (PVDF) membrane (Bio-Rad). The membrane was then blocked for 1-2 $\mathrm{h}$ at room temperature by incubating with $5 \%$ skim milk in phosphate-buffered saline (PBS) containing 0.1\% Tween-20. This was followed by incubation with a primary antibody for $30 \mathrm{~min}$ at room temperature. The specific bands were visualized by a further incubation with an HRP (horseradish peroxidase)-conjugated secondary antibody, followed by a chemiluminescence reaction using the ECL system (Amersham, Buckinghamshire, UK) according to the manufacturer's instructions. The rabbit polyclonal anti- $\beta$-actin antibody was used as a control and an anti-Histone $\mathrm{H} 1$ antibody was used as a control for the nuclear fraction.

The subcellular fractionation of cells. The cytoplasmic and the nuclear fractions were obtained by using a NE-PER kit (Pierce Biotechnology Inc, Rockford, IL) according to the manufacturer's protocol. The nuclear fraction was further extracted with $0.6 \mathrm{M} \mathrm{NaCl}$ containing $10 \mathrm{mM}$ Hepes, $5 \mathrm{mM}$ $\mathrm{MgCl}_{2}, 0.5 \%$ NP40, $1 \%$ Triton X-100, 1 mM PMSF, $10 \mu \mathrm{g} / \mathrm{ml}$ leupeptin and $10 \mu \mathrm{g} / \mathrm{ml}$ aprotinin.

Statistical analysis. Differences were analyzed using Student's t-test and $\mathrm{P}<0.05$ was considered significant. All experiments were performed at least three times. The data are presented as the mean \pm standard deviation $(\mathrm{SD})$.

\section{Results}

The suppression of TGF- $\beta 1$-induced Smad-dependent transcription by $\beta 1$-integrin. Since our previous study showed that the overexpression of $\beta 1$-integrin inhibited the TGF- $\beta 1$ induced growth suppression of HCC cells (16), we first examined whether or not $\$ 1$-integrin can alter the TGF- 31 induced Smad-dependent transcription. As shown in Fig. 1, TGF- $\$ 1$ increased the Smad-dependent transcription in HepG2 cells, but failed to increase the luciferase activity in the $\beta 1$ integrin-transfected cells, thus indicating that $\beta 1$-integrin mediated the inhibition of the Smad-dependent transcription.

The alteration of the phosphorylation status of the Smad proteins in the $\beta 1$-integrin transfectants. The phosphorylation status of Smad2 and Smad3 in both the C-terminal and the linker regions was examined in wild-type, mock- and B1integrin-transfected HepG2 cells under unstimulated conditions. Fig. 2 shows that the C-terminal region of Smad2 $(\mathrm{pSmad} 2 \mathrm{C})$ and $\mathrm{Smad} 3(\mathrm{pSmad} 3 \mathrm{C})$ remained unphosphorylated in the B1-integrin transfectants, in the wild-type cells and in the mock-transfected cells. However, the linker 


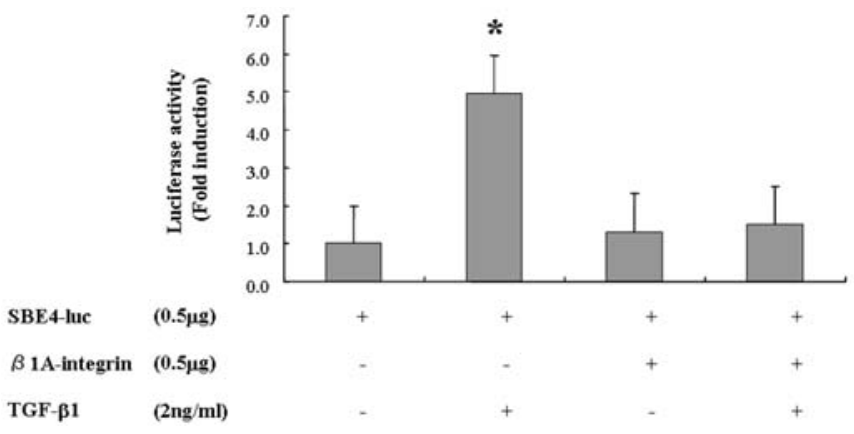

Figure 1. The inhibition of TGF-B1-induced Smad-dependent transcriptional activity by $\beta 1$-integrin. The HepG2 cells were transfected with the SBE4luciferase reporter gene plasmid with or without the 31 -integrin expression plasmid and were treated with $2 \mathrm{ng} / \mathrm{ml}$ of TGF- 31 for $24 \mathrm{~h}$. All experiments were performed in triplicates and data are shown as the mean fold induction of control with SD. * $\mathrm{P}<0.05$ compared with control cells without TGF- 31 .

region of Smad2 $(\mathrm{pSmad} 2 \mathrm{~L})$ and $\operatorname{Smad} 3(\mathrm{pSmad} 3 \mathrm{~L})$ in the B1-integrin transfectants were phosphorylated, but those in the wild-type and the mock-transfected cells remained unphosphorylated.

The modulation of the TGF- $\beta 1$-induced phosphorylation of Smad proteins in the $\beta 1$-integrin-transfected cells. The phosphorylation changes that occur after the TGF- 31 treatment were investigated (Fig. 3). In the wild-type and in the mocktransfected cells, $2 \mathrm{ng} / \mathrm{ml}$ of TGF- $\beta 1$ transiently phosphorylated the C-terminal region of Smad2 (pSmad2C) and Smad3 (pSmad3C), but did not induce phosphorylation in the linker region of Smad2 (pSmad2L) and Smad3 (pSmad3L). However, TGF- 31 could not induce the phosphorylation of the C-terminal region of Smad2 and Smad3 in the B1-integrin transfectants, even though it induced the phosphorylation in the linker region of Smad2. The phosphorylation in the linker region of Smad3 was not affected by TGF- $B 1$ in the wild-type, in the mock- and ß1-integrin-transfected cells.

The altered nuclear translocation of the phosphorylated form of Smad proteins in the $\beta 1$-integrin-transfected cells. The

p-Smad2-C
p-Smad2-L
t-Smad2
p-Smad3-C
p-Smad3-L
t-Smad3
$\beta$-actin

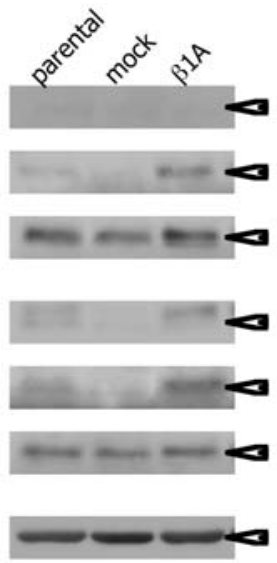

Figure 2. The expression and the phosphorylation status of Smad2 and Smad3 in the parental, the mock- and $B 1 \mathrm{~A}$-integrin-transfected HepG2 cells. The cells without TGF- 11 stimulation were analyzed by Western blotting with anti-Smad2 and anti-Smad3 antibodies. pSmad2C, Smad2 phosphorylated at C-terminal site; pSmad2L, Smad2 phosphorylated at the linker region; tSmad2, total Smad2; pSmad3C, Smad3 phosphorylated at the C-terminal site; pSmad3L, Smad3 phosphorylated at the linker region; tSmad3, total Smad3.

ability of the 31 -integrin to modulate the nuclear translocation of Smad proteins after the TGF- $\beta 1$ treatment was investigated (Fig. 4). The cells were separated into the cytoplasmic (Cy) and nuclear $(\mathrm{Nu})$ fractions with or without TGF-ß1. TGF-ß1 induced the phosphorylation of the C-terminal region of Smad2 and Smad3 and stimulated the nuclear accumulation of the C-terminal phosphorylated form of Smad2 and Smad3 in the wild-type and in the mock-transfected HepG2 cells. The linker region of Smad2 and Smad3 was not phosphorylated by TGF- $\$ 1$ and the accumulation of the linker-phosphorylated forms of Smad2 and Smad3 were not observed. In contrast, TGF- 31 could not induce C-terminal phosphorylation of Smad2 and Smad3 and did not stimulate the accumulation of the C-terminal phosphorylated form of Smad2 and Smad3 in the B1-inegrin-transfected cells. Although the phosphorylation of the linker region of Smad2 and Smad3 was not observed in the wild-type and in the mock-transfected cells, the linker

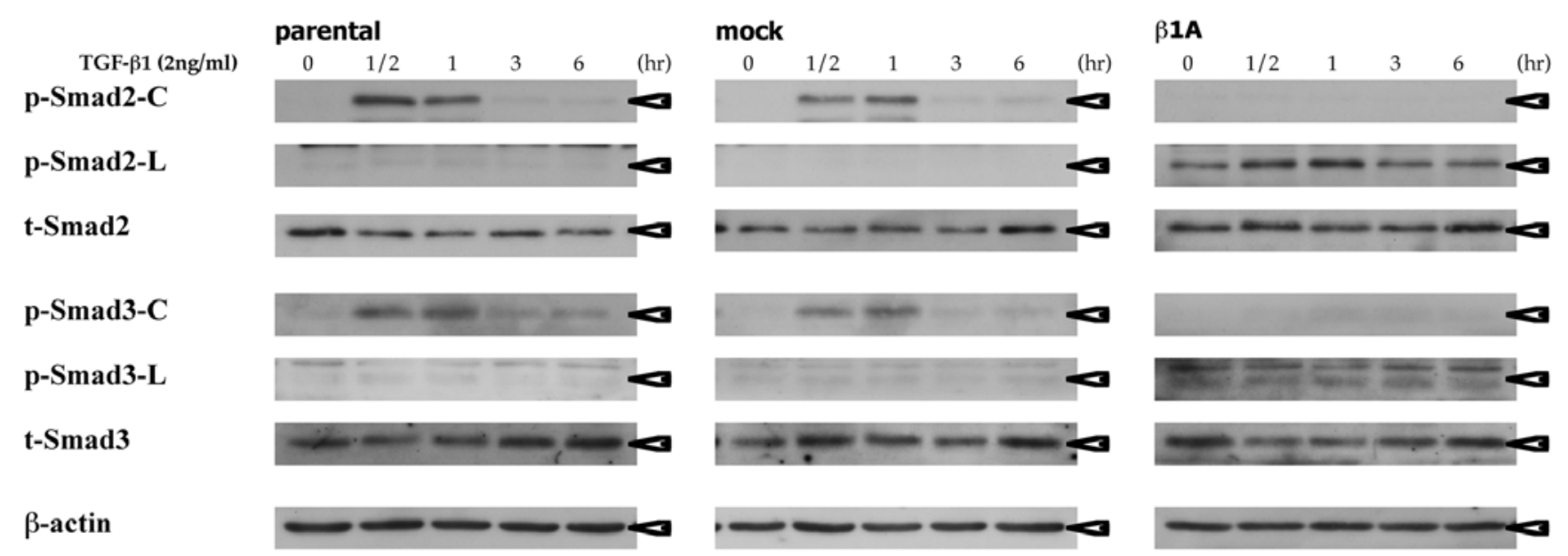

Figure 3. The modulation of the TGF- 31 -induced phosphorylation of the Smad proteins in the parental, the mock- and $ß 1 \mathrm{~A}$-integrin-transfected HepG2 cells. The cells were treated with $2 \mathrm{ng} / \mathrm{ml}$ of TGF- $\beta 1$, were harvested at the indicated times and were analyzed by Western blotting using each antibody. pSmad2C, Smad2 phosphorylated at C-terminal site; pSmad2L, Smad2 phosphorylated at the linker region; tSmad2, total Smad2; pSmad3C, Smad3 phosphorylated at the C-terminal site; pSmad3L, Smad3 phosphorylated at the linker region; tSmad3, total Smad3. 


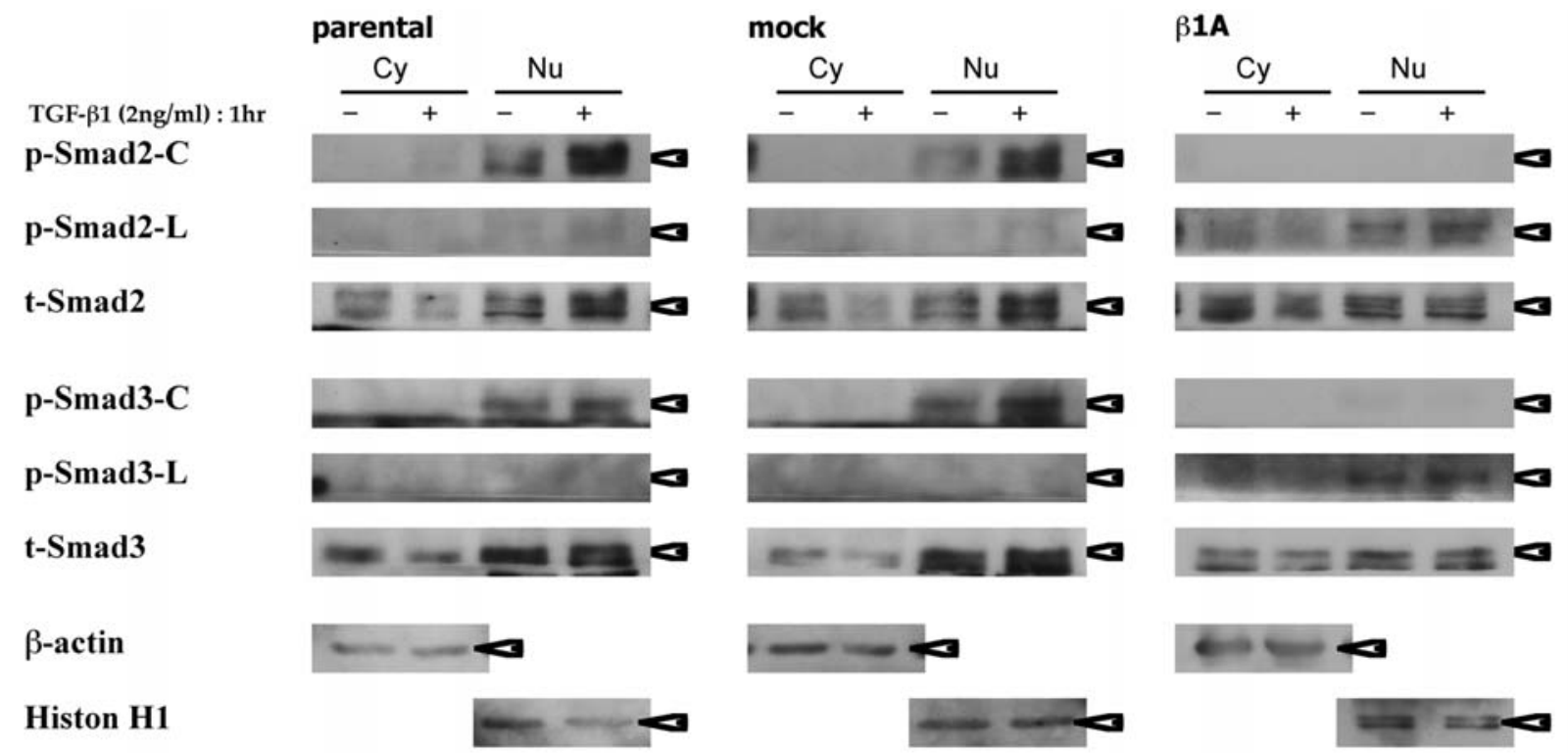

Figure 4. The altered nuclear translocation of the phosphorylated Smad proteins after TGF-ß1 treatment in B1A-integrin-transfected HepG2 cells. The cells were separated into the cytoplasmic $(\mathrm{Cy})$ and nuclear $(\mathrm{Nu})$ fractions with or without $2 \mathrm{ng} / \mathrm{ml}$ of TGF- $\beta 1$ for $1 \mathrm{~h}$ and were analyzed by Western blotting. Histone H1 was detected as the control for the nuclear proteins. pSmad2C, Smad2 phosphorylated at C-terminal site; pSmad2L, Smad2 phosphorylated at linker region; tSmad2, total Smad2; pSmad3C, Smad3 phosphorylated at the C-terminal site; pSmad3L, Smad3 phosphorylated at the linker region; tSmad3, total Smad3.

PD $98059(20 \mu \mathrm{M})$

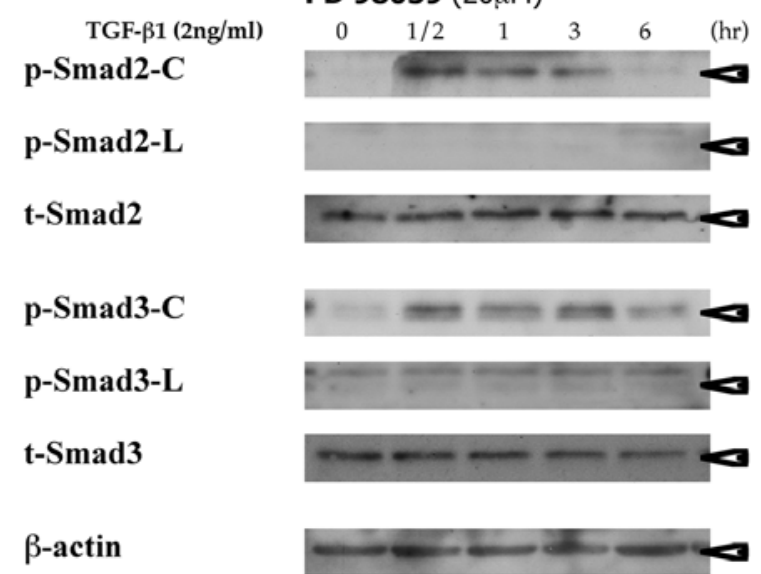
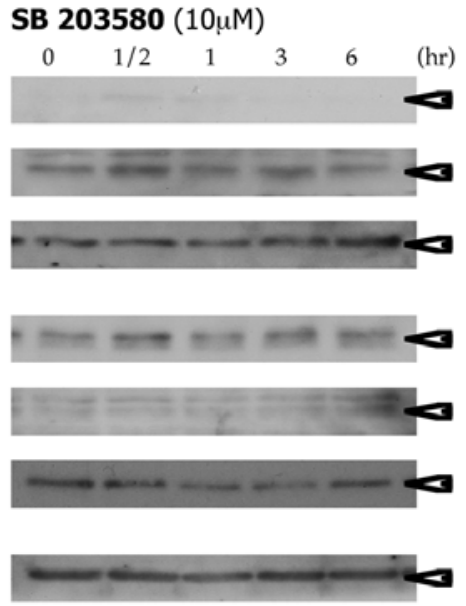

SP $600125(10 \mu \mathrm{M})$
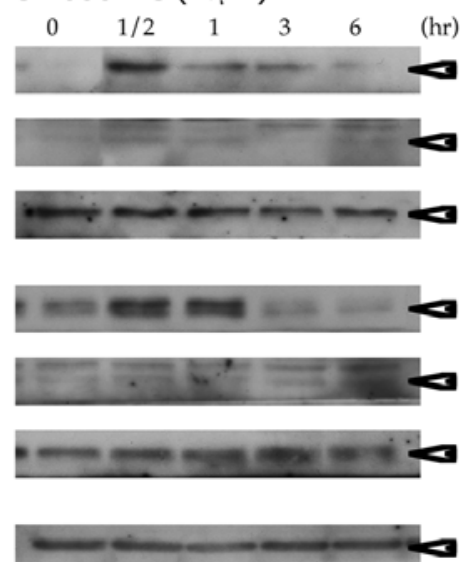

Figure 5. The involvement of the MAP kinase pathway in the alteration of the Smad phosphorylation in the B1A-integrin-transfected cells. The HepG2 cells overexpressing B1A-integrin were treated with PD98509 (ERK inhibitor; left), SB203580 (p38 inhibitor; center) and SP600125 (JNK inhibitor; right) for 6 h prior to the 1-h treatment with $2 \mathrm{ng} / \mathrm{ml}$ of TGF- $\beta 1$. pSmad2C, Smad2 phosphorylated at the C-terminal site; pSmad2L, Smad2 phosphorylated at the linker region; tSmad2, total Smad2; pSmad3C, Smad3 phosphorylated at the C-terminal site; pSmad3L, Smad3 phosphorylated at the linker region; tSmad3, total Smad3.

region of Smad2 and Smad3 was already phosphorylated in the B1-integrin-transfected cells at the basal level and the linker-phosphorylated form of Smad2 and Smad3 was present in the nucleus irrespective of the TGF- 31 stimulation.

The involvement of the MAP kinase pathway in the alterations of the TGF- $\beta 1$-regulated Smad phosphorylation by $\beta 1$-integrin. Since the ERK, the p38 and JNK pathways are involved in the alterations of the TGF- $\beta 1$-induced growth suppression in the 11 -integrin-transfected HCC cells (16), further experiments were conducted to determine whether or not the MAP kinase pathway is involved in the changes of the TGF- 31 -regulated Smad phosphorylation in $\beta 1$-integrin-transfected cells. The HepG2 cells, overexpressing $\$ 1$-integrin, were pretreated with
PD98509 (ERK inhibitor), SB203580 (p38 inhibitor) and SP600125 (JNK inhibitor), respectively. The pretreated cells were then treated with $2 \mathrm{ng} / \mathrm{ml}$ of TGF- 11 and were analyzed by Western blotting for changes in the phosphorylation state of the Smad proteins (Fig. 5). The treatment with the ERK and the JNK inhibitors restored the TGF- 31 -induced C-terminal phosphorylation of Smad2 that was impaired in the B1-integrin transfectants. However, the p38 inhibitor did not restore the TGF-31-induced phosphorylation of the C-terminal region of Smad2. Phosphorylation of the linker region of Smad2 was not affected by the ERK and the JNK inhibitors. In comparison, the p38 inhibitor induced the phosphorylation of the linker region of Smad2, indicating that the p38 pathway contributes to the suppression of the phosphorylation of the linker region 


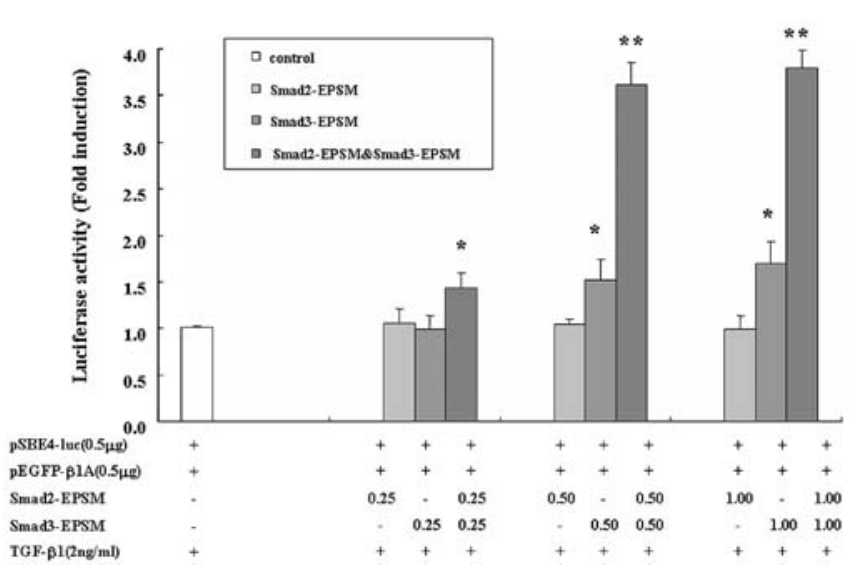

Figure 6. The restoration of the TGF-ß1-induced Smad-mediated transcription by mutant Smads lacking phosphorylation sites in the linker region. The HepG2 cells were transfected with the SBE4 reporter plasmid and the B1integrin expression plasmid together with mutant Smads lacking phosphorylation sites in the linker region (Smad2-EPSM and Smad3-EPSM) were then treated with $2 \mathrm{ng} / \mathrm{ml}$ of TGF- $\beta 1$ for $24 \mathrm{~h}$. The data are shown as the mean $\pm \mathrm{SD}$ from three independent experiments. ${ }^{*} \mathrm{P}<0.05,{ }^{* *} \mathrm{P}<0.01$ compared with control without Smad2-EPSM and Smad3-EPSM.

Table I. Effects of the ERK, p38 and JNK pathways on the phosphorylation of Smad proteins in the linker and C-terminal regions.

\begin{tabular}{lccc}
\hline Phosphorylation site of Smads & ERK & p38 & JNK \\
\hline pSmad2-C & $\downarrow$ & $\rightarrow$ & $\downarrow$ \\
pSmad2-L & $\uparrow$ & $\downarrow$ & $\uparrow$ \\
pSmad3-C & $\downarrow$ & $\downarrow$ & $\downarrow$ \\
pSmad3-L & $\uparrow$ & $\uparrow$ & $\uparrow$ \\
\hline
\end{tabular}

pSmad2C, Smad2 phosphorylated at C-terminal site; pSmad2L, Smad2 phosphorylated at linker region; pSmad3C, Smad3 phosphorylated at the C-terminal site; pSmad3L, Smad3 phosphorylated at the linker region; $\uparrow$, stimulation; $\downarrow$, inhibition; $\rightarrow$, equivocal.

of Smad2. The phosphorylation of the C-terminal site of Smad 3 by TGF- 31 was restored by all three inhibitors, but the phosphorylation of the linker region of Smad3 was suppressed by these inhibitors, suggesting that the ERK, the p38 and the JNK pathways all participated in the linker phosphorylation of Smad3 by $ß 1$-integrin.

The restoration of the TGF- $\beta 1$-induced Smad-dependent transcription by Smads mutated in the linker-region phosphorylation sites. Because the phosphorylation of the linker region of $S m a d 2 / 3$ appears to play important roles in the alteration of Smad signaling, we transfected the Smad2/3 plasmids (bearing mutations in the linker region and lacking phosphorylation sites) and tested whether or not the TGF-31induced Smad-dependent transcription was restored. As shown in Fig. 6, the transfection of the Smad3/ESPM partially restored the TGF- $ß 1$-induced Smad transcription. Although the Smad2/ESPM alone did not restore the TGF- 1 1-induced Smad transcription, the co-transfection of the Smad2/ESPM and the
Smad3/ESPM further increased the luciferase activity induced by TGF-ß1.

\section{Discussion}

TGF- $\beta$ is highly expressed in many malignant tumors, including HCC, with varying sensitivity of the tumor cells to TGF- $\beta$. The loss of the growth inhibitory effects of TGF- $\beta$ is regarded as an important pathway to carcinogenesis. The resistance to TGF- $\beta$ results from the repression of TßRI, TßRII, Smad2 and/or Smad4 (20). Previous studies showed that the linker region of the R-Smads can be phosphorylated by several kinases, and this linker-phosphorylated form responds to the TGF- 31 -induced growth inhibitory signal that is mediated by the phosphorylation of the C-terminal SSXS motif. The loss of the C-terminal phosphorylation of the R-Smads, which results in the repression of the anti-proliferative effects of TGF- $\beta$, is caused by the phosphorylation of the linker region of Smad2/3. The phosphorylation of the R-Smads are caused by an oncogenic Ras-mediated ERK MAP kinase activation (10) and/or by the cyclin-dependent kinases, CDK4 and CDK2 (14). The TGF- $\beta$ signaling is modulated by the growth factors and/or the inflammatory cytokines through JNK and/or p38 $(11,12)$. Chronic inflammation is closely linked to the development of many types of cancers, including HCC (21). Matsuzaki et al (22) recently reported that the phosphorylation at the linker region of the R-Smads, by the inflammatory cytokines acting through the JNK pathway, correlated with the loss of the phosphorylation at the Cterminal site of the R-Smads, thus leading to the promotion of hepatocarcinogenesis.

Experimental and clinical observations have suggested that liver fibrosis is a prerequisite for hepatocarcinogenesis (1-4). ß1-integrin, a major subunit of the heterodimeric receptor for ECM, plays important roles in carcinogenesis (23). Integrin serves as a survival factor by protecting the cells from various apoptosis-inducing factors, including TGF- $\beta$ (15-17). $ß 1$ integrin-induced activation of MAP kinase plays a critical role in protecting the $\mathrm{HCC}$ cells from the growth inhibition and apoptosis induced by TGF-B1 (16). However, the alterations of the Smad signaling by $\$ 1$-integrin in the HCC cells remain to be elucidated. This study demonstrated that the TGF- $\beta 1$ induced phosphorylation status of the R-Smads was altered in the B1-integrin-overexpressing HCC cells, and that the MAP kinases differentially contributed to the phosphorylation of the linker and to the C-terminal region of R-Smads (Table I). A specific feature of $\beta 1$-integrin-transfected cells is the loss of the phosphorylation of the C-terminal site in both Smad2 and Smad3 after TGF- 31 treatment. However, the linker region of Smad2 and Smad3 is constitutively phosphorylated in the ß1-integrin-transfected cells. In accordance with these results, Matsuzaki et al (22) observed an increased phosphorylation at the linker region of $S \operatorname{mad} 3$ in the hepatocytes adjacent to the fibrotic areas in liver that were chronically infected with the hepatitis $\mathrm{C}$ virus.

In this study, the differential phosphorylation of Smad2 and Smad3 was observed when the cells were treated with a p38 inhibitor. Although the p38 inhibitor increased the phosphorylation of the Smad2 linker region in the B1-integrintransfected HCC cells, the phosphorylation state of the Smad3 
linker region remained unchanged. Smad3 plays an essential role in the TGF- $\beta$-mediated signal transduction that leads to the suppression of cell growth and the reduction in $\mathrm{HCC}$ development (24). The activation of p38 MAP kinase was required for the Smad3-mediated suppression of HCC development. However, it is not clear if Smad2, Smad3 or both proteins are responsible for the TGF- 3 -induced EMT, cell motility and invasion. Ju et al showed that Smad2-deleted hepatocytes were sensitive to TGF- $\beta$-induced growth arrest, apoptosis and EMT, but that the Smad3-deleted hepatocytes were not (25). In addition, the Smad2-deleted hepatocytes spontaneously acquired the characteristic features of the EMT, thus suggesting that Smad2 suppressed the hepatocyte growth and the EMT, independent of the TGF- $\beta$ signaling. Therefore, elucidating the mechanism by which Smad2 and Smad3 regulate the TGF- $\beta$ signaling pathway is important for the development of a better strategy for cancer therapy.

Because the alterations in the TGF- $\beta$ signaling pathway play an important role in promoting carcinogenesis, the TGF- $\beta$ signaling appears to be an attractive target for cancer prevention and therapy. However, TGF- $\beta$ plays a tumorpromoting and tumor-suppressive role, therefore, shutting down the TGF- $\beta$ signaling may be unfavorable because it may lead to the suppression of TGF-3-mediated growth inhibition. Hence, caution should be taken when the TGF- $\beta$ signal is targeted for inhibiton because blockage of TGF- $\beta$ does not always lead to the inhibition of cancer development and progression.

With respect to the tumor-promoting action of the linkerphosphorylated form of R-Smad and the tumor-suppressing action of the C-terminal phosphorylated form, the selective phosphorylation of the C-terminal site and/or the selective inhibition of the linker region of R-Smads might be ideal processes for the restoration of TGF- $B$-mediated tumorsuppressive action. Recently, protein phosphatase 1A, a magnesium-dependent $\alpha$-protein phosphatase 2C $\alpha$ (PPM1A/ $\mathrm{PP} 2 \mathrm{Ca}$ ), was reported to dephosphorylate the $\mathrm{C}$-terminal region of Smad2/3 and to act as a negative regulator of the TGF- $\beta$ induced signals (26). This suggests that PPM1A inhibition might restore the growth-inhibitory action of TGF- 3 .

The phosphorylation of the linker region in the R-Smads, especially in Smad3, seems to play an important role in shifting the TGF- $\beta$ signaling from a tumor-suppressive to an oncogenic state by increasing the invasion and the metastatic potential through the EMT (27). Since the MAP kinase pathway and the CDKs are involved in the phosphorylation of the linker region in the R-Smads, the inhibitors of these kinases might be useful for the recovery of the TGF- $\beta$-mediated tumor-suppressive signaling. Recently, the dephosphorylation and/or the blockade of phosphorylation in the linker region of Smad2 and/or Smad3 were shown to restore the altered TGF- $\beta$ signaling (28-30). Therefore, the specific inhibition of the phosphorylation of the linker-region might provide a more selective and effective approach for the prevention and the treatment of cancers.

\section{References}

1. Okuda K: Hepatocellular carcinoma. J Hepatol 32: 225-237, 2000.

2. LIovet JM, Burroughs A and Bruix J: Hepatocellular carcinoma. Lancet 362: 1907-1917, 2003
3. Sakaida I, Hironaka K, Uchida K, Suzuki C, Kayano K and Okita K: Fibrosis accelerates the development of enzyme-altered lesions in the rat liver. Hepatology 28: 1247-1252, 1998.

4. Omata M and Shiratori Y: Long-term effects of interferon therapy on histology and development of hepatocellular carcinoma in hepatitis C. J Gastroenterol Hepatol (Suppl 15): E134-E140, 2000.

5. Friedman SL: The cellular basis of hepatic fibrosis. Mechanisms and treatment strategies. N Engl J Med 328: 1828-1835, 1993.

6. Moses HL, Yang EY and Pietenpol JA: TGF- $\beta$ stimulation and inhibition of cell proliferation: new mechanistic insights. Cell 63: 245-247, 1990

7. Zavadil $\mathrm{J}$ and Bottinger EP: TGF- $\beta$ and epithelial-to-mesenchymal transitions. Oncogene 24: 5764-5774, 2005.

8. Heldin $\mathrm{CH}$, Miyazono K and ten Dijke P: TGF- $\beta$ signaling from cell membrane to nucleus through SMAD proteins. Nature 390: 465-471, 1997.

9. Massague J, Seonae J and Wotton D: Smad transcription factors. Genes Dev 19: 2783-2810, 2005.

10. Kretzschmar M, Doody J, Timokhina I and Massague J: A mechanism of repression of TGFß/Smad signaling by oncogenic Ras. Genes Dev 13: 804-816, 1999.

11. Mori S, Matsuzaki K, Yoshida K, Furukawa F, Tahashi Y, Yamagata H, Sekimoto G, Seki T, Matsui H, Nishizawa M, Fujisawa $\mathrm{J}$ and Okazaki K: TGF-beta and HGF transmit the signals through JNK-dependent $\mathrm{Smad} 2 / 3$ phosphorylation at linker regions. Oncogene 23: 7416-7429, 2004.

12. Furukawa F, Matsuzaki K, Mori S, Tahashi Y, Yoshida K, Sungano Y, Yamagata H, Matsushita M, Seki T, Inagaki Y, Nishizawa M, Fujisawa J and Inoue K: p38 MAPK mediates fibrogenic signal through Smad3 phosphorylation in rat myofibroblasts. Hepatology 38: 879-889, 2003.

13. Matsuura I, Denissova NG, Wang G, He D, Long J and Liu F: Cyclin-dependent kinases regulated the antiproliferative function of Smads. Nature 430: 226-231, 2004.

14. Hynes RO: Integrins: versatility, modulation, and signaling in cell adhesion. Cell 69: 11-25, 1992.

15. Sethi T, Rintoul RC, Moore SM, MacKinnon AC, Salter D, Choo C, Chilvers ER, Dransfield I, Donnely SC, Strieter R and Haslett C: Extracellular matrix proteins protect small cell lung cancer cells against apoptosis: a mechanism for small cell lung cancer growth and drug resistance in vivo. Nat Med 5: 662-668, 1999.

16. Zhang H, Ozaki I, Mizuta T, Yoshimura T, Matsuhashi S, Eguchi Y, Yasutake T, Hisatomi A, Sakai T and Yamamoto K: Transforming growth factor- $\$ 1$-induced apoptosis is blocked by B1-integrin-mediated mitogen-activated protein kinase activation in human hepatoma cells. Cancer Sci 95: 878-886, 2004.

17. Zhang H, Ozaki I, Mizuta T, Matsuhashi S, Yoshimura T, Hisatomi A, Tadano J, Sakai T and Yamamoto K: Beta1-integrin protects hepatoma cells from chemotherapy-induced apoptosis via a mitogen-activated protein-kinase-dependent pathway. Cancer 95: 896-906, 2002.

18. Zhang H, Ozaki I, Mizuta T, Yoshimura T, Matsuhashi S, Hisatomi A, Tadano J, Sakai T and Yamamoto K: Mechanism of B1-integrin-mediated hepatoma cell growth involves p27 and S-phase kinase-associated protein 2. Hepatology 38: 305-313, 2003.

19. Zawel L, Dai JL, Buckhaults P, Zhou S, Kinzer KW, Vogelstein B and Kern SE: Human Smad3 and Smad4 are sequence-specific transcription activators. Mol Cell 1: 611-617, 1998.

20. Levy L and Hill CS: Alterations in components of the TGF-beta superfamily signaling pathways in human cancer. Cytokine Growth Factor Rev 17: 41-58, 2006.

21. Karin M, Lawrence T and Nizet V: Innate immunity gone awry linking microbial infections to chronic inflammation and cancer. Cell 124: 823-835, 2006.

22. Matsuzaki K, Murata M, Yoshida K, Sekimoto G, Uemura Y, Sakaida N, Kaibori M, Kamiyama Y, Nishizawa M, Fujisawa J, Okazaki K and Seki T: Chronic inflammation associated with hepatitis $\mathrm{C}$ virus infection perturbs hepatic transforming growth factor $\beta$ signaling, promoting cirrhosis and hepatocellular carcinoma. Hepatology 46: 48-57, 2007.

23. Hanahan D and Weinberg RA: The hallmarks of cancer. Cell 100: 57-70, 2000.

24. Yang Y, Zhang G, Feigenbaum L and Zhang YE: Smad3 reduces susceptibility to hepatocellular carcinoma by sensitizing hepatocytes to apoptosis through downregulation of Bcl-2. Cancer Cell 9: 445-447, 2006. 
25. Ju W, Ogawa A, Heyer J, Nierhof D, Yu L, Kucherlapati R, Shafritz DA and Bottinger EP: Deletion of Smad2 in mouse liver reveals novel functions in hepatocytes growth and differentiation. Mol Cell Biol 26: 654-667, 2006.

26. Lin X, Duan X, Liang Y, Su Y, Wrighton KH, Long J, Hu M, Davis CM, Wang J, Brunicardi FC, Shi Y, Chen Y, Meng A and Feng X: PPM1A functions as a Smad phosphatases to terminate TGF- $\beta$ signaling. Cell 125: 915-928, 2006.

27. Matsuzaki K: Smad3 phosphoisoform mediated signaling during sporadic human colorectal carcinogenesis. Histol Histopathol 21: 645-662, 2006.

28. Wrighton KH, Willis D, Long J, Liu F, Lin X and Feng X Small C-terminal phosphatases dephosphorylate the regulatory linker region of Smad2 and Smad3 to enhance transforming growth factor- $\beta$ signaling. J Biol Chem 281: 38365-38375, 2006
29. Sapkota G, Knockarert M, Alarcon C, Montalvo E, Brivanlou AH and Massague J: Dephsophorylation of the linker regions of Smad 1 and Samd2/3 by small C-terminal domain phosphatases has distinct outcomes for bone morphogenetic protein and transforming growth factor- $\beta$ pathways. J Biol Chem 281: 40412-40419, 2006.

30. Sekimoto G, Matsuzaki K, Yoshida K, Mori S, Murata M, Seki T, Matsui H, Fujisawa J and Okazaki K: Reversible Smaddependent signaling between tumor suppression and oncogenesis. Cancer Res 67: 5090-5096, 2007. 